\title{
BMJ Open Depressive symptoms in people with vision impairment: a cross-sectional study to identify who is most at risk
}

\author{
Claire Nollett, ${ }^{1}$ Barbara Ryan, ${ }^{2}$ Nathan Bray, ${ }^{3}$ Catey Bunce, ${ }^{4}$ Robin Casten, ${ }^{5}$ \\ Rhiannon Tudor Edwards, ${ }^{3}$ David Gillespie, ${ }^{1}$ Daniel J Smith, ${ }^{6}$ Miles Stanford, ${ }^{7}$ \\ Tom H Margrain ${ }^{2}$
}

To cite: Nollett C, Ryan $B$, Bray N, et al. Depressive symptoms in people with vision impairment: a crosssectional study to identify who is most at risk. BMJ Open 2019;9:e026163. doi:10.1136/ bmjopen-2018-026163

- Prepublication history for this paper is available online. To view these files, please visit the journal online (http://dx.doi. org/10.1136/bmjopen-2018026163).

Received 20 August 2018 Revised 26 November 2018 Accepted 27 November 2018

A) Check for updates

(C) Author(s) (or their employer(s)) 2019. Re-use permitted under CC BY-NC. No commercial re-use. See rights and permissions. Published by BMJ.

For numbered affiliations see end of article.

Correspondence to

Claire Nollett;

nollettcl@cardiff.ac.uk

\section{ABSTRACT}

Objective To identify the risk factors for significant depressive symptoms in people with visual impairment in England and Wales to provide information on who is most at risk and to whom support services could be targeted in future.

Design A cross-sectional study using baseline data from a pragmatic randomised controlled trial.

Setting and participants 990 participants aged 18 or over attending 1 of 14 low-vision rehabilitation primary care optometry-based clinics in South Wales or two hospital clinics in London.

Outcome measure A score of $\geq 6$ on the Geriatric Depression Scale-15 was classed as clinically significant depressive symptoms.

Results In a multivariable logistic regression model, significant depressive symptoms were associated with age (adjusted OR (AOR) $=0.82,95 \% \mathrm{Cl}: 0.66$ to $0.90, \mathrm{p}<0.001$ ), ethnicity (AOR non-white compared with white $=1.72$, $95 \% \mathrm{Cl}: 1.05$ to $2.81, \mathrm{p}=0.031$ ), total number of eye conditions (AOR for two vs one condition $=0.98,95 \% \mathrm{Cl}$ : 0.67 to 1.43 ; three or more vs one condition $=0.34,95 \% \mathrm{Cl}$ : 0.15 to $0.75, p=0.026$ ), self-reported health (AOR for excellent vs poor $=0.01,95 \% \mathrm{Cl}: 0.00$ to 0.12 ; very good vs poor $=0.06,95 \% \mathrm{Cl}$ : 0.03 to 0.13 ; good vs poor $=0.14$, $95 \% \mathrm{Cl}: 0.08$ to 0.24 ; fair vs poor $=0.28,95 \% \mathrm{Cl}: 0.18$ to $0.46, \mathrm{p}<0.001$ ) and self-reported visual functioning ( $A O R=1.45,95 \% \mathrm{Cl}: 1.31$ to $1.61, \mathrm{p}<0.001$ ).

Conclusion Younger age, a non-white ethnicity, fewer eye conditions and poorer self-reported health and visual function are risk factors for significant depressive symptoms in this population.

Trial registration number ISRCTN46824140; Pre-results.

\section{BACKGROUND}

Vision impairment impacts on all aspects of life and is associated with reduced functional ability, falls, social isolation and reduced quality-of-life. ${ }^{1-3}$ There is also a growing awareness that it has a negative impact on mental health status too. Population-based studies provide robust evidence of an association between vision impairment and depression. Typically those with a vision impairment are 2-3 times more likely to be depressed. ${ }^{45}$ In Britain,

\section{Strengths and limitations of the study}

- This is the first study of risk factors for depressive symptoms in people seeking help for vision impairment in England and Wales.

- It benefits from a large sample size $(\mathrm{n}=990)$ and a high response rate $(n=990 / 1323,74.8 \%)$ which increase the generalisability of the findings.

- It examines factors which can be readily assessed by practitioners in primary care and general hospital clinics who come into contact with people with vision impairment, enabling them to be alerted to those most at risk and in need of signposting to support services.

- However, it excludes some more difficult to measure factors, such as vision-specific distress, coping style and perceived social support which may also predict depression in this population.

- The study uses a cross-sectional design, so conclusions about direction of causality cannot be made.

for example, a large survey of $>13000$ older adults found that the prevalence of significant depressive symptoms in those with good vision living in the community was about $4.6 \%$ while in those with a vision impairment $(<6 / 18)$ it was $13.5 \% .^{5}$

The prevalence of significant depressive symptoms is also high in those accessing rehabilitation services. Results from a study in Australia found that when screened with the two item Patient Health Questionnaire-2, $37 \%$ of patients attending rehabilitation clinics or eye care services screened positive for depressive symptoms. ${ }^{6}$ Here in the UK, we screened over 1000 patients attending low-vision rehabilitation appointments using the Geriatric Depression Scale (GDS-15), as part of the Depression in Visual Impairment Trial (DEPVIT), a randomised controlled trial. ${ }^{7}$ We found that $43 \%$ of patients reported significant depressive symptoms (score $\geq 6$ ) and, significantly, $74.8 \%$ were not receiving any treatment for depression. ${ }^{8}$ This finding 
supports previous reports that people with a vision impairment are less likely to have their depression identified than those with good vision. ${ }^{9-11}$

Because depression goes underdetected in this patient group, there is a need to improve routine screening. The National Institute for Health and Care Excellence (NICE), in their guidelines on 'Depression in adults with a chronic physical health problem, ${ }^{, 12}$ suggest that practitioners working in primary care and general hospital clinics should be aware that patients with a chronic physical health problem, especially those with functional impairment, are at a high risk of depression. They recommend being alert to possible depression and asking two simple screening questions to detect depression. ${ }^{12}$ Those screening positively should be referred to an appropriate professional for assessment, in most cases, the patient's general practitioner (GP). Screening should occur in both low-vision-specific settings such as rehabilitation clinics, and in primary care and general clinical settings such as diabetes or stroke clinics, where vision impairment is prevalent. In busy primary care and general clinics, understanding who is most at risk of depression among this patient group using easy to determine factors may help clinicians to target depression screening and signpost patients to supportive services.

Previous cross-sectional studies of patients from outpatient eye clinics and low-vision rehabilitation services have identified several risk factors for depression including: being female, being relatively younger in age, living alone and having lower acceptance of vision loss, ${ }^{13}$ reporting poorer self-reported health and having a history of mental health problems, ${ }^{13}{ }^{14}$ reporting poorer vision-specific functioning, higher levels of vision-specific distress, having an avoidant coping style and lower perceived adequacy of social support. ${ }^{14}$ A longitudinal prospective cohort study of 540 patients from outpatient low-vision organisations in the Netherlands and Belgium found that people who developed depressive symptoms over a 2-year period were more likely to be: living alone, having just enough money to cover their expenses, have macular degeneration, have problems with adaptation to vision loss, have reduced health related quality of life and be experiencing symptoms of anxiety. ${ }^{15}$

The above studies were conducted in the Netherlands, Belgium and Australia, and we do not know if the same risk factors apply to a British population. Therefore, it is useful to examine the risk factors for depressive symptoms in people with sight loss in England and Wales, using a large sample of consecutive attendees to services. The findings will enable clinicians in primary care and general hospital clinics to allocate resources to screening those most at risk.

The aim of this study was to identify the risk factors for significant depressive symptoms in people with vision impairment attending rehabilitation clinics in England and Wales using baseline data from a randomised controlled trial of interventions for depression (DEPVIT). ${ }^{7}$ We focused our examination mainly on characteristics which can be easily identified in routine practice, for example, age and ethnicity, to provide a straightforward approach to identifying high-risk patients based on readily available information.

\section{METHODS}

\section{Study design and participants}

This cross-sectional study was undertaken as part of DEPVIT. ${ }^{7}$ Eligible participants were consecutive adult patients who were seeking help for vision impairment at specialist visual rehabilitation services taking part in DEPVIT. Fourteen primary care-based rehabilitation services recruited participants in South Wales. Services were readily accessible high street practices, accepted self-referral and tended to cater to older adults with age-related eye conditions living in the local community. A secondary care rehabilitation clinic based at Guys and St Thomas' Hospital and a National Health Service (NHS) outreach clinic providing low-vision services in Southwark recruited participants from the London area. Access to these two specialist clinics was by referral only. All consecutive attendees aged 18 or over were considered eligible for the study, unless they lived outside the catchment area for the trial or if they had previously been screened for depression as part of the study (some people had more than one appointment during the length of the study, but we only wanted to screen them and invite them to take part once). The study adhered to the Declaration of Helsinki.

\section{Funding and public and Patient involvement}

The study was funded by Guide Dogs, a voluntary sector organisation who work closely with people with vision impairment and understand their experiences and preferences. They carried out a review prior to funding to ensure the research questions were relevant and the study design appropriate. Patients with a vision impairment reviewed and provided feedback on the depression questionnaire. Patients were not involved in the recruitment to or conduct of the study.

\section{Measures}

\section{Depression}

GDS- $15^{16}$ is one of the most widely used instruments for the screening of depression in older adults. The questionnaire has 15 questions, and the completion time is approximately $5 \mathrm{~min}$. Possible scores range from 0 to 15 , with higher scores indicating a greater number of depressive symptoms. We chose to use dichotomous categories rather than the continuous scale as this reflects the scale's use in clinical practice as a screening tool to identify those who warrant further investigation. We used the conventional scoring approach rather than Rasch analysis to facilitate direct comparison with published studies and to facilitate clinically valid results. A score of 6 or more was taken to be indicative of significant depressive symptoms. ${ }^{5}$ 


\section{Risk factors}

We recorded gender, age, ethnicity (white, Asian/Asian British, black/black British or other), physical illness (number and type from a list of seven plus an 'other' category) and ocular diagnosis (number and type of eye conditions from a list of five plus an 'other' category), factors which would be readily available to clinicians working with people with sight loss and have been considered in previous studies.

We also measured self-reported general health as this has consistently been shown to be a risk factor for depression $^{613-15}$ and can be easily measured using a single item question from the SF-12, 'In general, would you say your overall health is: excellent, very good, good, fair or poor?' The question has had widespread use as a single-item measure, including in previous studies of visual impairment and depression ${ }^{514}$ and has shown to be significantly and independently associated with specific health problems, use of health services, changes in functional status, recovery from episodes of ill health, mortality and sociodemographic characteristics of respondents. ${ }^{17}$

To provide information on vision related factors for low-vision practitioners who have access to this information, we also measured presenting corrected binocular visual acuity using Early Treatment Diabetic Retinopathy Study (ETDRS) LogMAR and recorded time since vision loss in years. As previous studies have found no evidence of an association between objective measures of visual acuity and depression, ${ }^{13-15}$ we were interested to see whether a subjective measure of visual function would be associated. Self-reported visual functioning was measured using the 7-item National Eye Institute Visual Function Questionnaire (NEI-VFQ 7) which includes a subset of questions from the NEI-VFQ that have previously been shown to be responsive to rehabilitation service intervention. ${ }^{18}$ As the NEI-VFQ 7 is commonly reported in the published literature with Rasch analysis, we transformed the Likert responses using the Rasch derived scoring key provided by Ryan $e t a l^{18}$ to calculate a score for each completed questionnaire. A higher score indicates a greater perceived difficulty with visual functioning. Questionnaires with three or more missing items were counted as missing and excluded from the analysis.

\section{Procedures}

Participants who were eligible to take part in the study were sent a questionnaire in large print format containing the GDS-15, NEI-VFQ 7 and single-item question about health, along with their appointment letter at least 1 week before their low-vision assessment. They were asked to complete the questionnaire at home, with assistance if needed, and to bring it along to their appointment. Those who did not return a completed questionnaire were given the opportunity to complete another copy at the clinic, before their appointment. The low-vision practitioner reviewed the participant's responses with them at the start of the assessment and asked for their written consent to use their anonymised responses in the study. For those who consented, information on gender, date of birth, ethnicity, physical illness, ocular diagnosis, corrected ETDRS LogMAR acuity and time since vision loss first identified were recorded on a Case Report Form (CRF). Those who screened positive for depressive symptoms (GDS-15 score of $\geq 6$ ) were offered entry to the DEPVIT trial if eligible, or a referral to their GP if not eligible. ${ }^{19}$

CRFs completed by the clinicians were sent to the research coordinating centre at Cardiff University by secure fax where the validity and completeness of the data were checked. Any missing or out of range data were queried with the practitioner and checked with clinical notes. Five per cent of all CRFs and surveys were double entered. The error rate was less than $2 \%$ and identified errors were corrected. The number of eligible patients who did not complete the survey and the number who did not consent for their data to be used for research purposes were also recorded. The final dataset was then locked and transferred to the statistical team for analysis. The descriptive statistics were tabulated using SPSS V.23, and the regression models were fitted using STATA V.13.1.

\section{Statistical analysis}

Participant characteristics were summarised for those with significant depressive symptoms (GDS-15 $\geq 6$ ) and those without (GDS-15<6). Categorical variables were summarised as numbers and percentages and continuous variables as medians with interquartile ranges. In all cases we report the number of participants for whom data were missing.

Where the GDS-15 was not fully completed, completed answers were totalled to give a final score provided that the number of questions not answered was two or less. ${ }^{5}$ If three or more questions were unanswered, the GDS-15 data were regarded as missing and the participant excluded from the analysis.

Logistic regression was used to determine the independent relationship between each of the potential risk factors and significant depressive symptoms. The potential risk factors were initially included individually (univariable analysis) and then entered into a multivariable logistic regression analyses in blocks to determine which variables remain associated with significant depressive symptoms after controlling for the other factors. The events-per-variable was sufficient to allow inclusion of all potential risk factors, so no selection was required. ${ }^{20}$ However, due to co-linearity, it was not possible to include both number and type of physical illnesses or both number and type of eye conditions. Therefore, a decision was made to include only number of illnesses and eye conditions, as it was hypothesised that burden of multiple diagnoses would be more important than type of diagnosis: those with multiple morbidity are at twice the risk of depression than those without multiple morbidity. ${ }^{21}$ The variables were entered into the analysis in blocks, starting with the risk factors which could be most easily identified in routine clinics, and ending with those requiring more 
time or adaptation to practice to assess. The blocks were: (1) demographics (gender/age/ethnicity); (2) demographics and physical health (number of illnesses); (3) demographics, physical health and eye health (number of eye conditions/visual acuity/time since vision loss); (4) demographics, physical health, eye health and self-report measures (self-report health/visual functioning). We calculated the area under the Receiver Operating Characteristic (ROC) curve to quantify the overall ability of each (additional) block of variables to correctly discriminate between those with and without depression.

\section{RESULTS}

A total of 1323 consecutive adult patients attended the low-vision rehabilitation clinics during the 30 month recruitment period. Of these, 312 were not screened for depression because the practitioner felt it was inappropriate at the time (because the patient was too ill, had dementia or was recently bereaved); or the patient had forgotten to complete the questionnaire and there was no time at the assessment; or they did not consent for their data to be used for research. An additional 21 patients had three or more missing items on the GDS-15 and were excluded, leaving a final sample size of 990 and a complete response rate of $74.8 \%$. The median age of the participants was 79.0 years $(\mathrm{IQR}=66.0-85.0), 62.2 \%$ were female $(n=616)$ and $85 \%$ were white $(n=842)$. The overall prevalence of significant depressive symptoms was $42.5 \%$. This varies very slightly from our previously reported study $(43 \%)^{8}$ due to the methodology used in this study to calculate the total GDS-15 score (excluding those with $\geq 3$ missing items).

Tables 1-4 outline the demographic characteristics of the participants, their physical health measures, eye health measures and self-report health and vision measures respectively, split by those with and without significant depressive symptoms. They also summarise the results of the univariable logistic regression using ORs presented with $95 \%$ CIs and $p$ values. The variables ethnicity, number of physical illnesses and number of eye conditions had a small number of participants in some categories, hence the categories were collapsed before being entered into the regression analysis. Both the original and collapsed categories are presented in the tables. Table 5 Summarises the results of the multivariable logistic regression using adjusted ORs (AOR) and are presented with $95 \%$ CIs and $p$ values.

\section{Demographics}

Table 1 shows that those with a higher prevalence of significant depressive symptoms were male, younger or non-white. In the univariable analysis, age and ethnicity were associated with significant depressive symptoms. An increase in age was associated with lower odds of participants having depression and having ethnicity other than white was associated with higher odds of having depression. These variables remain associated once other variables were controlled for in the multivariable analysis final model (table 5). There was no evidence of an association between gender and significant depressive symptoms.

\section{Physical health}

The prevalence of depression was lowest in those with no physical illness $(29.8 \%)$ and highest in those with three or more illnesses $(54.3 \%$, table 2). In the univariable analysis, an increase in the number of physical illnesses was associated with higher odds of having significant depressive symptoms. This association remained when controlling for demographics and eye health but was no longer associated when controlling for subjective health and visual function.

\section{Eye health}

Those with a higher prevalence of depression had one eye condition, worse visual acuity or less time since vision loss (table 3). The univariable analysis found no evidence of an association between significant depressive symptoms and number of eye conditions, visual acuity and time since vision loss. However, when controlling for other factors in the final model, an increase in the number of eye conditions was associated with lower odds of having significant depressive symptoms.

\section{Self-report measures}

The prevalence of depression was highest in those with poor self-rated health $(81.5 \%)$ and lowest in those with excellent health $(4.3 \%)$. Those with significant depressive symptoms had worse self-rated visual functioning (table 4). Worse self-rated health and visual functioning were associated with higher odds of having significant depressive symptoms in both the univariable analysis and multivariable final model.

The area under the ROC curve was 0.59 when demographics alone were entered into the model, increasing to 0.65 when physical and eye health variables were considered and reaching 0.81 when self-report measures were added.

\section{DISCUSSION}

This study identified the risk factors for significant depressive symptoms in people with vision impairment attending vision rehabilitation clinics in England and Wales. We focused mainly on risk factors which can be easily identified in primary care and general hospital clinics, so as to provide a pragmatic approach to identifying high risk patients. To inform ophthalmic clinicians who may have access to more detailed information on eye health, we also included a range of vision related variables. Our findings showed that among older adults, those of relatively younger age, with an ethnicity other than white and poorer self-reported health and visual function had higher odds of having significant depressive symptoms. Number of physical illnesses was an 


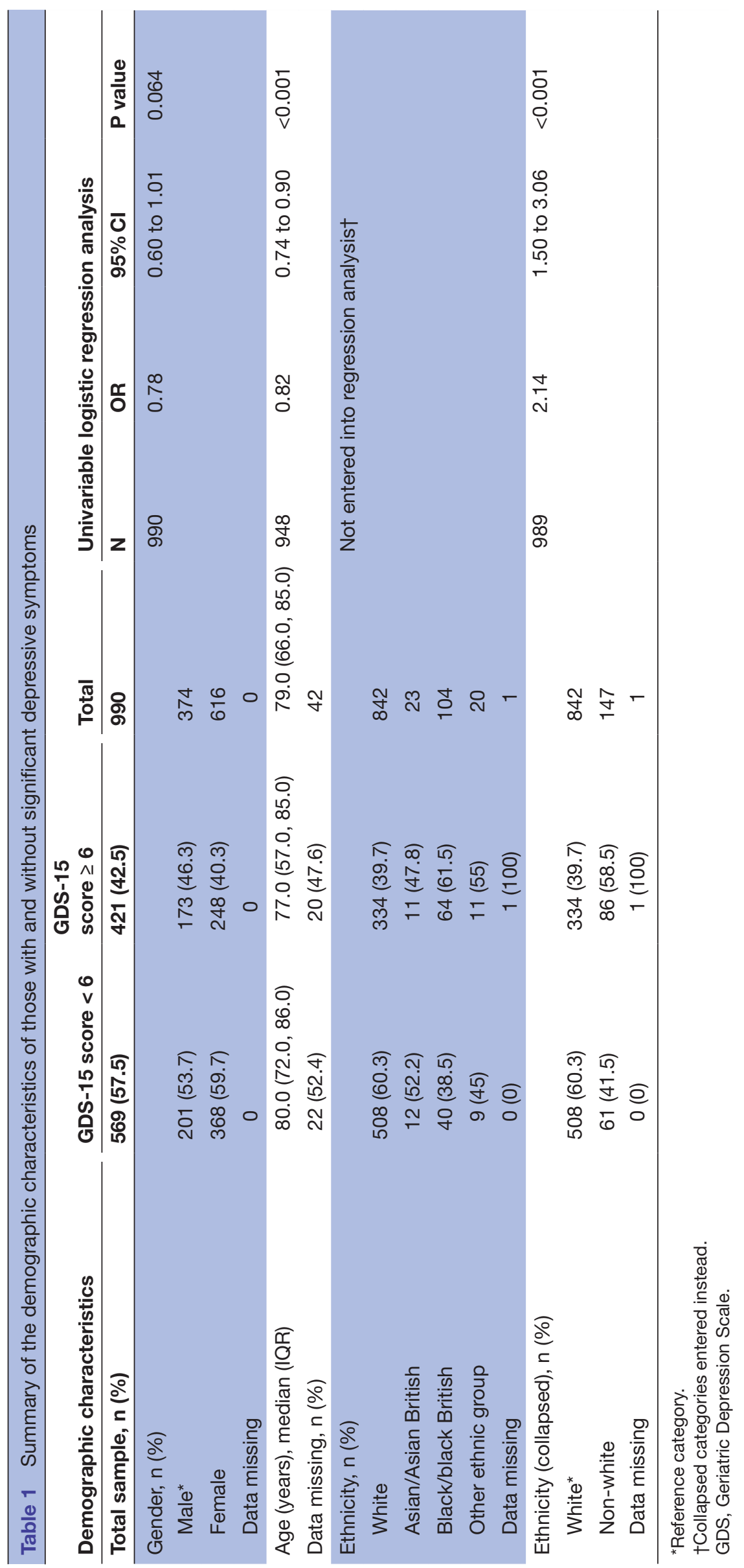


Table 2 Summary of the physical health of those with and without significant depressive symptoms

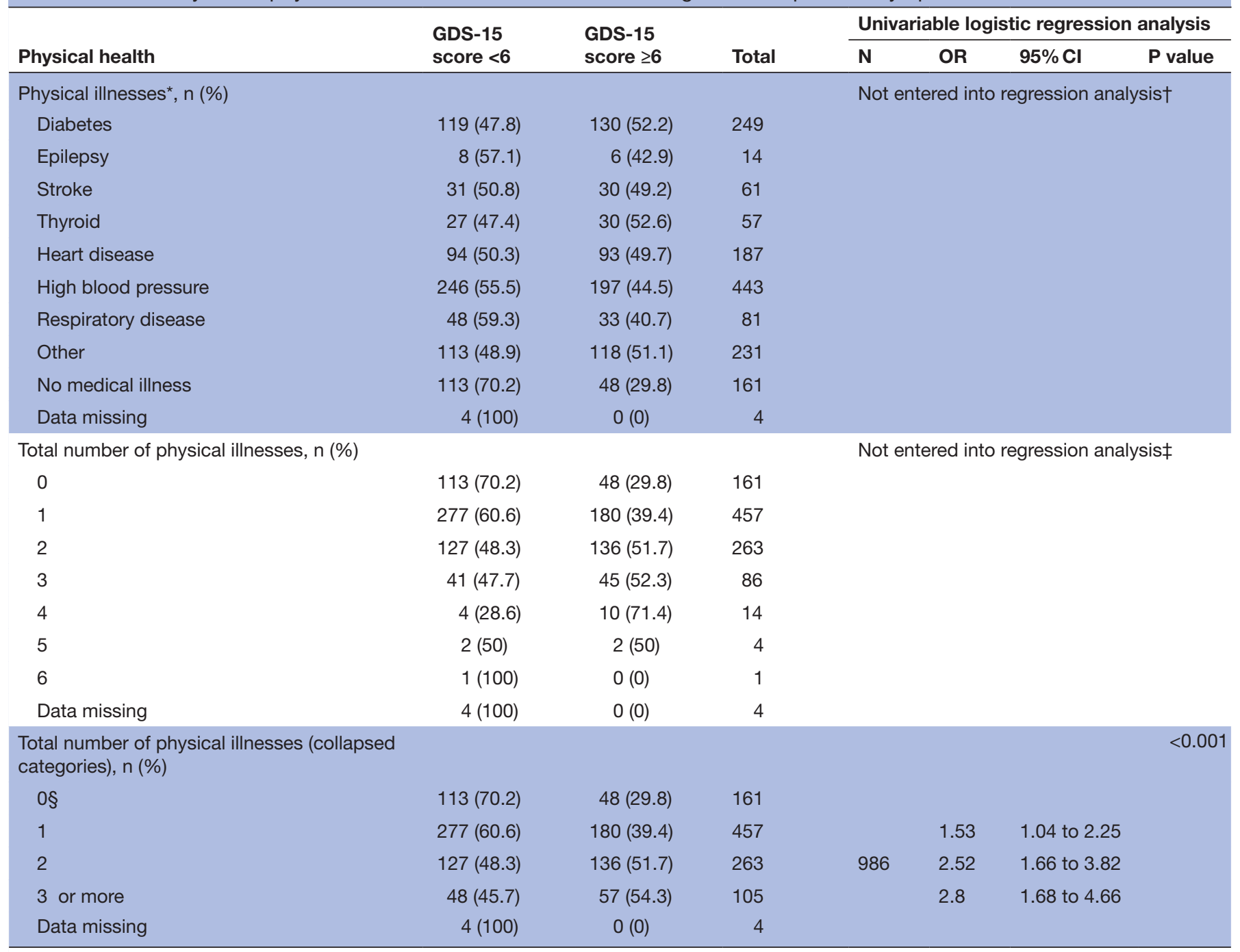

*Participants may have had more than one physical illness.

†Not entered due to high correlation with number of physical illnesses.

¥Collapsed categories entered instead.

§Reference category.

GDS, Geriatric Depression Scale.

independent predictor of depressive symptoms, but there was no evidence of an association when controlling for subjective health and vision function. The number of eye conditions was not an independent predictor of depressive symptoms, but was related to depression when other variables were controlled: less number of eye conditions was associated with higher odds. There was no evidence that gender, time since vision loss and visual acuity were associated with depression.

With regard to demographic factors, our findings demonstrate some support for, and discrepancies with, previous studies. In a study with an Australian population, ${ }^{14}$ a univariate analysis provided evidence that younger age was associated with depressive symptoms, and in a European and Australian sample (relatively) younger age was shown to be associated with subthreshold depression in a multivariable analysis. ${ }^{13}$ Our study corroborates these findings in a UK sample. This perhaps reflects the finding in the general population that people aged 40-59 years have higher rates of depression than those aged $\geq 60$ years $^{22}$ and those in middle-age have the highest risk. ${ }^{23}$ The reasons for this are not clearly understood, but one theory is that by mid-life, individuals have learnt to adapt to their strengths and weaknesses, and in mid-life 'quell their infeasible aspirations' ${ }^{23}$ In those with vision loss, being affected in middle-age rather than old age may add to this sense of lost aspirations and could also result in more restriction in life including difficulties in finding and staying in work, playing sport and so on. Our research found no evidence of an association between gender and depressive symptoms. Previous studies examining this association have differed in their findings. An Australian study showed no association in a univariate analysis, ${ }^{14}$ while a model with a European and Australian sample found being female was a predictor of subthreshold depression. ${ }^{13}$ The authors of a study with 


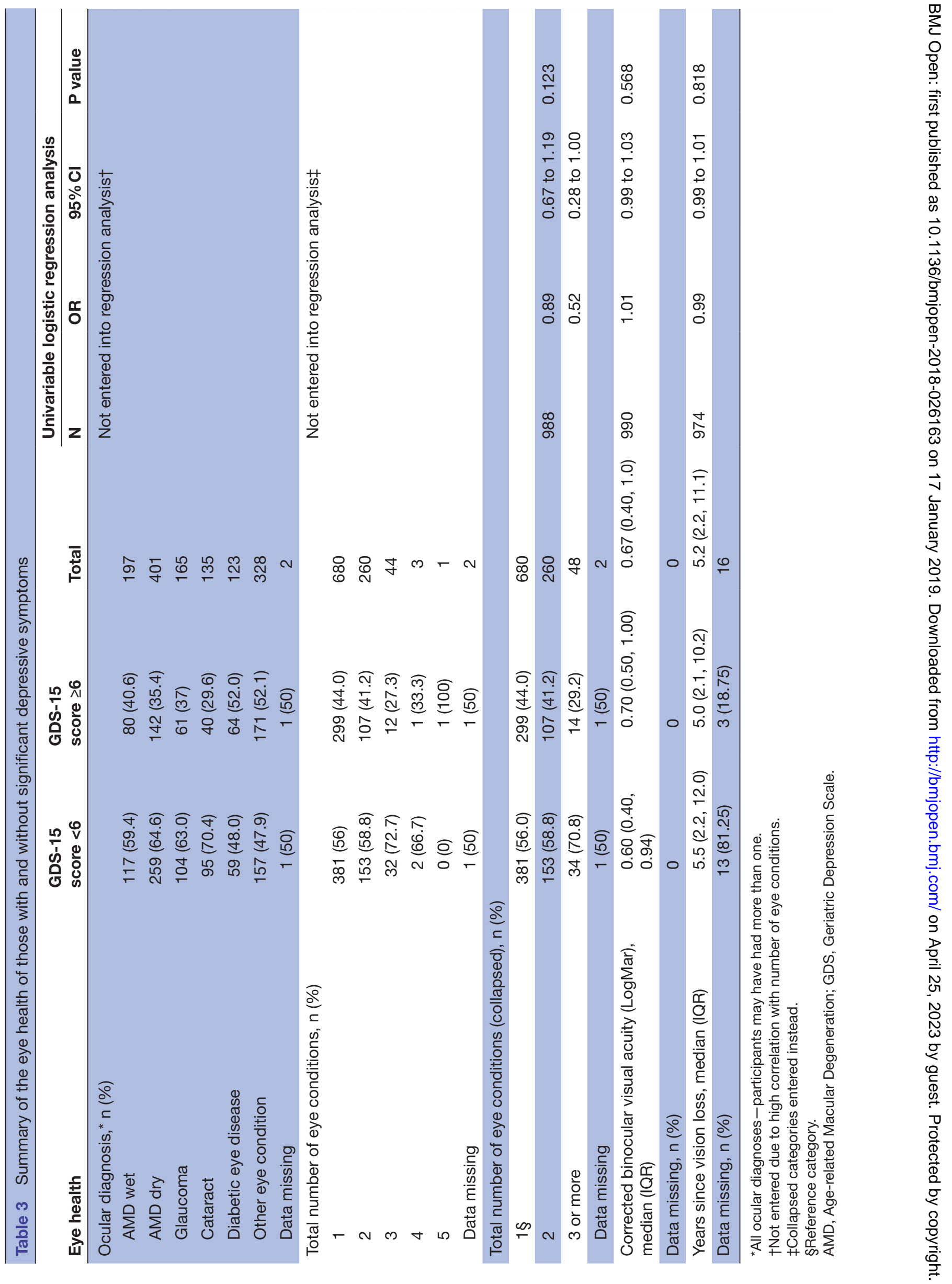


Table 4 Summary of the self-reported health and visual function of those with and without significant depressive symptoms

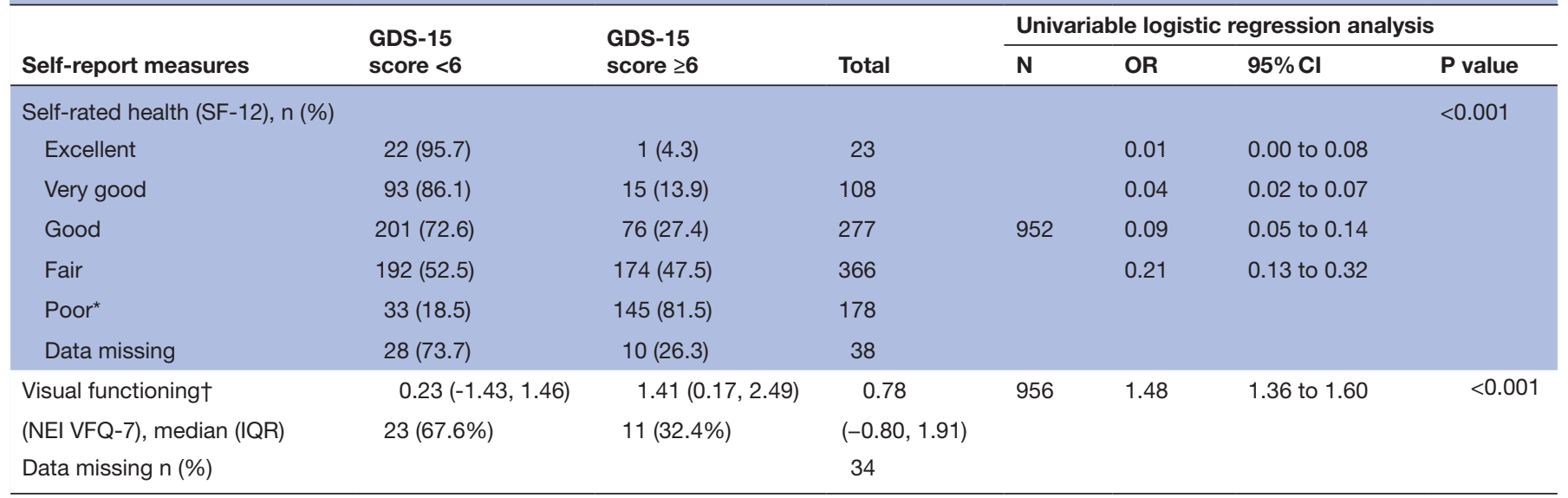

${ }^{*}$ Reference category.

†NEI VFQ-7 scores have been Rasch analysed and a higher score indicates a greater perceived difficulty with visual functioning.

GDS, Geriatric Depression Scale; NEI VFQ-7, 7-item National Eye Institute Visual Function Questionnaire.

Dutch and Belgian participants reported that their findings on gender were inconclusive. ${ }^{15}$ Differences in findings across the studies may indicate this factor is country specific, or may be due to differences in the measures used to assess depression. For example, we included people with all levels of depressive symptoms, whereas the European/Australian study included only subthreshold depression. It may be that being female is associated with subthreshold depression but there is no association when all levels of severity are considered. We found that having an ethnicity other than white was a risk factor. Recent studies on vision impairment and depression have not measured ethnicity, however an earlier study conducted in New Zealand found that ethnicity was not related to depression. ${ }^{24}$ Differences between that study and ours may be due to the different populations, with a wider variation in ethnicities in the UK and London in particular. The New Zealand study only recorded 'New Zealand born European' or 'other'. Therefore, future studies should include ethnicity as a variable to provide further clarification.

There is more consistency between European and Australasian studies and our UK study in terms of health. We demonstrated that those with poorer self-reported health were at much higher risk of depressive symptoms. This confirms previous research in vision-impaired people which has shown that poorer perceived health status, ${ }^{13}$ poorer self-reported health ${ }^{14}$ and poorer health related quality of life $\mathrm{e}^{24}$ are all predictors of depression. This is not surprising as patients may include their emotional health in a question about general health. Our study also found that a higher number of physical illnesses was an independent risk factor for depression. This is in line with findings from the non-vision-impaired population. A recent meta-analysis found a substantial relationship between multimorbidity (the presence of two or more chronic physical illnesses) and depression, reporting that people with multimorbidity are at twice the risk of depression to those without multimorbidity, and nearly three times at risk compared with those with no chronic physical condition. ${ }^{21}$ The authors suggest the relationship is bidirectional and cite the Activity Restriction Model of Depressed Affect ${ }^{25}$ which explains that multimorbidity contributes significantly to depressive symptoms through having to give up valued activities due to physical limitations. In our sample, the limitations of conditions such as stroke and diabetes may have compounded any mobility and functional issues already caused by sight loss which can make self-care, engaging in hobbies and getting out and about more difficult.

In terms of vision related factors, it is logical to assume that the chances of having depressive symptoms increases as visual acuity decreases. However, in line with other European and Australian studies, ${ }^{13-15}$ the results of the regression analysis do not support this hypothesis. What seems to be more important is self-reported visual function: those with worse self-reported visual function are more at risk of depressive symptoms. ${ }^{26}$ Therefore, clinicians should take care not to make assumptions about the likelihood of depression in only those with the lowest levels of vision as assessed by visual acuity. The relationship is likely to be bidirectional, with poorer visual function leading to loss of valued activities and mood, while lowered mood may influence a person's perception of their vision function. As with previous studies, ${ }^{13} 15$ time since the vision loss was first identified was also not a predictor of depression, indicating that patients may develop symptoms at any point on their sight loss journey. The more surprising finding was that people with three or more eye conditions had lower odds of having significant depressive symptoms than those with just one eye condition. On consultation with the literature, we suggest this finding may be explained in terms of acceptance: lower acceptance of vision loss has shown to be a predictor of subthreshold depression. ${ }^{13}$ In their work with people with diabetic eye disease and partial sight loss (some of whom also had glaucoma), Oehler-Giarratana and Fitzgerald report that patients described being in a state of 'limbo' where they 


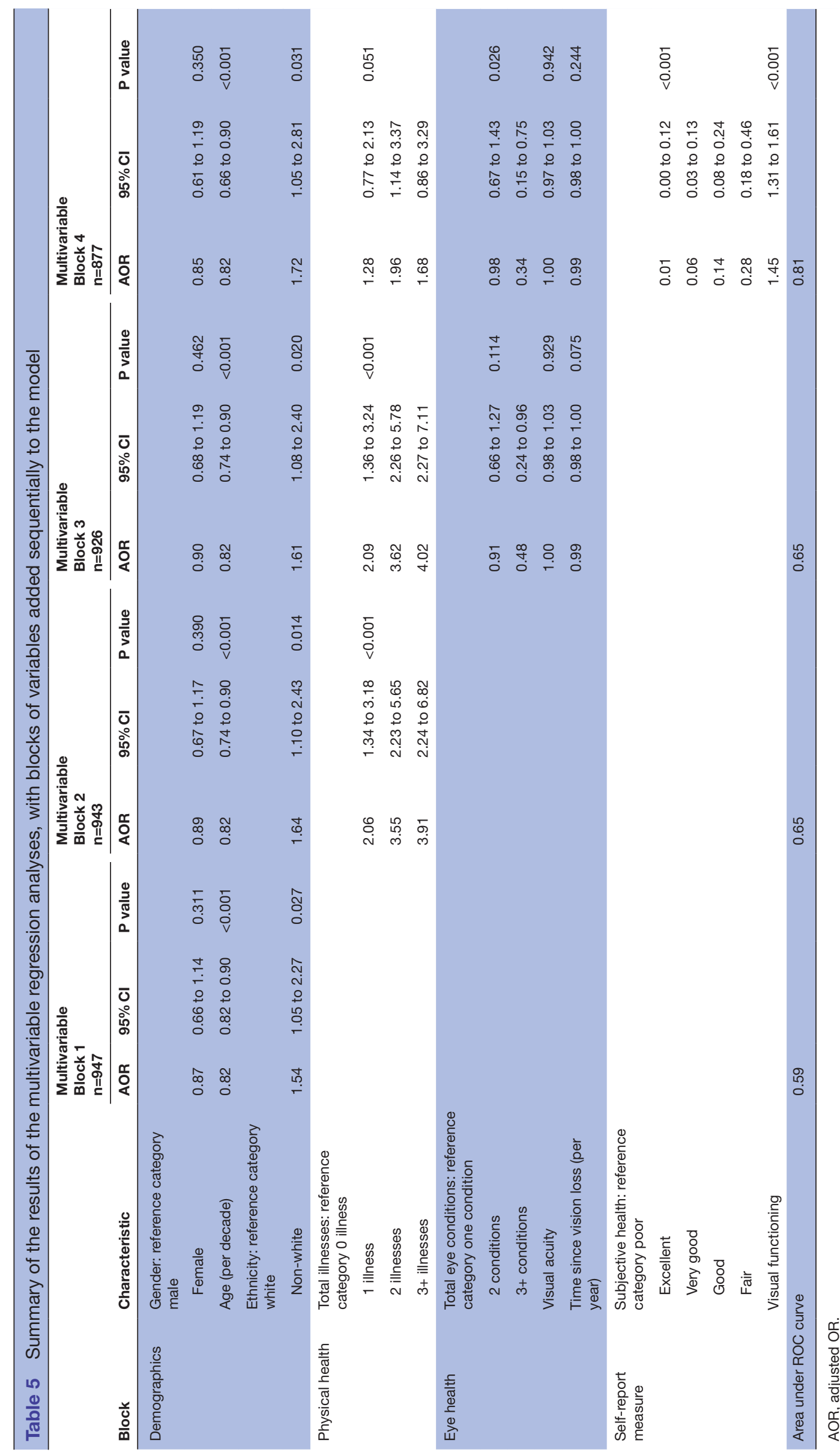

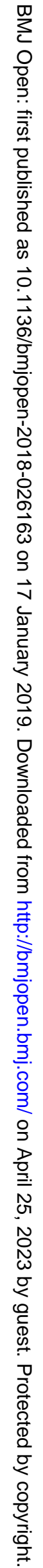


experienced uncertainty, fear and hope that vision might improve. ${ }^{27}$ Perhaps surprisingly, they expressed the view that total loss of vision would be a relief, as they could proceed through a healing phase and make plans for their future care. In our study, it is possible that those with three or more eye conditions had come to terms with the likelihood of further vision loss and reached a point of acceptance, whereas those with one eye condition were in the 'limbo' phase, with the hope that sight may improve but the fear that it might deteriorate, and therefore not reached this point of acceptance thus increasing their risk of depressive symptoms. None of the studies referred to in our introduction included number of eye conditions as a risk factor, and we could not find any studies which included both number of eye conditions and level of acceptance. Therefore, further research is needed to better understand this finding and possible explanation. Our research suggests that not all of the factors related to depressive symptoms in people with vision impairment are specific to that particular population. As with the general population, age, ethnicity and health are associated with risk of depression and this needs to be taken into consideration when understanding the link between vision impairment and depression, and when considering suitable interventions.

This research added to the literature by examining risk factors in a British sample of people with vision impairment. The study benefited from a large sample size and a high response rate, enhancing the generalisability of the findings. As we included 14 low-vision rehabilitation clinics across primary and secondary care, we believe the findings are transferrable to both settings in the UK. Our study employed validated measures of depressive symptoms and incorporated risk factors which are easy to identify in primary care and hospital clinics. Therefore, the results can be easily integrated in clinical practice to target screening.

However, inevitably there were some value judgements in how we chose our criteria for selecting the range of potential factors in our study. This means that other parameters which have previously been shown to be predictors of depression, for example, vision-specific distress, lower perceived adequacy of social support and avoidant coping, ${ }^{14}$ were not measured and therefore cannot be included in the risk profile advice to clinicians. These parameters can only be assessed using additional questionnaires which would have increased the overall response burden in the study, and furthermore, it is unlikely that these variables would be measured in routine practice and therefore were not within the scope of our study.

We chose to dichotomise the GDS-15 to reflect how it would be used in practice, as a screening tool for identifying patients who would benefit from screening in clinic and potentially signposting to support services. However, we acknowledge that this may have led to a reduction in power and loss of information. ${ }^{28} \mathrm{~A}$ further limitation of the study is the use of a cross-sectional design which means conclusions about direction of causality are not possible. Finally, while the completion rate of the GDS-15 was high, a number of patients were not screened at the discretion of the practitioners, including because they felt the patient was too ill, had dementia or had recently been bereaved, or they did not consent for their answers be used for research. Therefore, there may be a risk of bias as the non-completers may be systematically different from those that completed the questionnaire and consented to their data being used. Similarly, we excluded cases with missing data from the multivariable analysis and this simple approach to missing data may have introduced some bias. However, as only 113/990 (11\%) were excluded, the risk of bias was low.

For the first time, for a population in England and Wales, our study demonstrates that for patients with vision impairment, there are several risk factors for depression which can be easily identified by those coming in to contact with people with sight loss. We recommend that all clinicians working with people with sight loss are alert to these factors. We advise screening higher risk patients using the simple two question screen recommended in the NICE guidelines. ${ }^{12}$ If a patient is identified as having likely depression, they should be managed according to the guidelines which includes referral to an appropriate professional, for example, the GP. Local pathways should be established to manage this referral. However, because the prevalence of depressive symptoms is so high in low-vision clinics, we recommend that low-vision practitioners introduce depression screening as part of routine care with all patients.

Future research could include qualitative work to clarify the pathway from the risk factors identified here to the onset of depression, to aid the development of interventions for depression in this population.

\section{Author affiliations}

${ }^{1}$ Centre for Trials Research, College of Biomedical and Life Sciences, Cardiff University, Cardiff, UK

${ }^{2}$ School of Optometry and Vision Sciences, Cardiff University, Cardiff, UK ${ }^{3}$ Centre for Health Economics and Medicines Evaluation, Bangor University, Bangor, UK

${ }^{4}$ School of Population and Environmental Sciences, Kings College London, London, UK

${ }^{5}$ Department of Psychiatry and Human Behavior, Thomas Jefferson University, Philadelphia, Pennsylvania, USA

${ }^{6}$ Institute of Health and Wellbeing, University of Glasgow, Glasgow, UK ${ }^{7}$ Medical Eye Unit, St Thomas' Hospital, London, UK

Acknowledgements The authors wish to thank the following for data collection: Sandra Jumbe, Rowanne Black, Kathy Davies, Denise Banford, lan Cousins, Huw Bellamy, Norma Davies, Ceri Goodman, Sharon Beatty, Lisa Evans, Leighton Griffiths, Matthew Harvey, David Jenkins, Jane Savage, Marek Karas, John Butler, Seema Rajoria, Rajiv Raja, Jane De Courcy, Ahalya Subramanian, Sonal Rughani and Sarah Janikoun.

Contributors TM, DJS, RTE and MS conceived the idea for the study and acquired funding. CN project-managed the study, supervised data collection, performed data cleaning, statistical analysis and wrote the first draft of the manuscript. CB and BR substantially contributed to the design of the study and to the acquisition of data. NB and RC contributed to the design of the study. DG provided statistical advice and supervision. All authors contributed to the design of the protocol, critically reviewed the manuscript for important intellectual content and approved the final manuscript. 
Funding The work was supported by Guide Dogs grant number OR2009-07b.

Competing interests None declared.

Patient consent for publication Not required.

Ethics approval The NHS South East Wales Research Ethics Committee Panel B approved this study.

Provenance and peer review Not commissioned; externally peer reviewed.

Data sharing statement There are no additional unpublished data from this study.

Open access This is an open access article distributed in accordance with the Creative Commons Attribution Non Commercial (CC BY-NC 4.0) license, which permits others to distribute, remix, adapt, build upon this work non-commercially, and license their derivative works on different terms, provided the original work is properly cited, appropriate credit is given, any changes made indicated, and the use is non-commercial. See: http://creativecommons.org/licenses/by-nc/4.0/.

\section{REFERENCES}

1. Christ SL, Zheng DD, Swenor BK, et al. Longitudinal relationships among visual acuity, daily functional status, and mortality: the Salisbury Eye Evaluation Study. JAMA Ophthalmol 2014;132:1400-6.

2. Court H, McLean G, Guthrie B, et al. Visual impairment is associated with physical and mental comorbidities in older adults: a crosssectional study. BMC Med 2014;12:181.

3. Dargent-Molina P, Favier F, Grandjean H, et al. Fall-related factors and risk of hip fracture: the EPIDOS prospective study. Lancet 1996;348:145-9.

4. Chou KL. Combined effect of vision and hearing impairment on depression in older adults: evidence from the English Longitudinal Study of Ageing. J Affect Disord 2008;106(1-2):191-6.

5. Evans JR, Fletcher AE, Wormald RP. Depression and anxiety in visually impaired older people. Ophthalmology 2007;114:283-8

6. Holloway EE, Sturrock BA, Lamoureux EL, et al. Depression screening among older adults attending low-vision rehabilitation and eye-care services: Characteristics of those who screen positive and client acceptability of screening. Australas J Ageing 2015;34:229-34.

7. Nollett CL, Bray N, Bunce C, et al. Depression in Visual Impairment Trial (DEPVIT): a randomized clinical trial of depression treatments in people with low vision. Invest Ophthalmol Vis Sci 2016;57:4247-54.

8. Nollett CL, Bray N, Bunce C, et al. High prevalence of untreated depression in patients accessing low-vision services. Ophthalmology 2016;123:440-1.

9. Crawford MJ, Prince M, Menezes P, et al. The recognition and treatment of depression in older people in primary care. Int J Geriatr Psychiatry 1998;13:172-6.

10. MacDonald AJ. Do general practitioners "miss" depression in elderly patients? Br Med J 1986;292:1365-7.

11. O'Connor DW, Rosewarne R, Bruce A. Depression in primary care. 2: general practitioners' recognition of major depression in elderly patients. Int Psychogeriatr 2001;13:367-74.
12. National Institute for Health and Care Excellence. Depression in adults with a chronic physical health problem: recognition and management. Clinical guideline [CG91]. 2009.

13. van der Aa HP, Xie J, Rees G, et al. Validated prediction model of depression in visually impaired older adults. Ophthalmology 2016;123:1164-6.

14. Rees G, Xie J, Holloway EE, et al. Identifying distinct risk factors for vision-specific distress and depressive symptoms in people with vision impairment. Invest Ophthalmol Vis Sci 2013;54:7431-8.

15. Heesterbeek TJ, van der Aa HPA, van Rens G, et al. The incidence and predictors of depressive and anxiety symptoms in older adults with vision impairment: a longitudinal prospective cohort study. Ophthalmic Physiol Opt 2017;37:385-98.

16. Sheikh JI, Yesavage JA. Geriatric Depression Scale (GDS): Recent evidence and development of a shorter version. Clinical Gerontologist: The Journal of Aging and Mental Health 1986;5:165-73.

17. Bowling A. Just one question: If one question works, why ask several? J Epidemiol Community Health 2005;59:342-5.

18. Ryan B, Court H, Margrain TH. Measuring low vision service outcomes: rasch analysis of the seven-item national eye institute visual function questionnaire. Optom Vis Sci 2008;85:112-21.

19. Margrain TH, Nollett C, Shearn J, et al. The Depression in Visual Impairment Trial (DEPVIT): trial design and protocol. BMC Psychiatry 2012;12:57.

20. Heinze G, Wallisch C, Dunkler D. Variable selection - a review and recommendations for the practicing statistician. Biom $J$ 2018;60:431-49.

21. Read JR, Sharpe L, Modini M, et al. Multimorbidity and depression: a systematic review and meta-analysis. J Affect Disord 2017;221:36-46.

22. Centre for Disease Control and Prevention. QuickStats: Prevalence of Current Depression* Among Persons Aged $\geq 12$ Years, by Age Group and Sex - United States, National Health and Nutrition Examination Survey, 2007-2010. Secondary QuickStats: Prevalence of Current Depression* Among Persons Aged $\geq 12$ Years, by Age Group and Sex - United States, National Health and Nutrition Examination Survey, 2007-2010 2012. 2012 https://www.cdc.gov/mmwr/preview/ $\mathrm{mmwrhtml} / \mathrm{mm6051a7.htm} \mathrm{(Accessed} 21$ Jun 2018).

23. Blanchflower DG, Oswald AJ. Is well-being U-shaped over the life cycle? Soc Sci Med 2008;66:1733-49.

24. Hayman KJ, Kerse NM, La Grow SJ, et al. Depression in older people: visual impairment and subjective ratings of health. Optom Vis Sci 2007;84:1024-30.

25. Williamson G, Shaffer D. The Activity restriction Model of Depressed Affect. In: Williamson G, Shaffer D, Parmelee P, eds. Physical illness and depression in older adults: Kluwer Academic Publishers, 2000.

26. Tabrett DR, Latham K. Factors influencing self-reported vision-related activity limitation in the visually impaired. Invest Ophthalmol Vis Sci 2011:52:5293-302.

27. Oehler-Giarratana J, Fitzgerald RG. Group therapy with blind diabetics. Arch Gen Psychiatry 1980;37:463-7.

28. Altman DG, Royston P. Statistics notes - the cost of dichotomising continuous variables. British Medical Journal 2006;332:1080-80. 